\title{
OBLIGACIÓN ÉTICA Y JURÍDICA DE PREVENIR Y REDUCIR LA APARICIÓN DE NUEVAS DISCAPACIDADES EN PERSONAS MAYORES EN CHILE
}

\begin{abstract}
Carolina Riveros Ferrada ${ }^{1}$, Rodrigo Barcia Lehmann ${ }^{2}$
Resumen: En este trabajo pretendemos evidenciar la incongruencia entre la reciente Ley № 20.584 con la Convención de Naciones Unidas sobre Derechos de las Personas con Discapacidad. Dichas personas, en determinado estadio de su enfermedad, no podrán participar de investigaciones en su calidad de discapacitados. Estimamos que esta imposibilidad no se condice con el artículo 25 de la Convención, que exige a los Estados prevenir y reducir al máximo la aparición de nuevas discapacidades, incluidas las que ocurren en personas mayores. La problemática que se genera no solo posee una perspectiva legal, sino que también produce un conflicto ético que afecta al Estado, a los profesionales médicos, a los pacientes y sus familiares. Por ello, proponemos una interpretación armónica y sistemática del artículo 28 de la Ley $\mathrm{N}^{\circ} 20.548$ con la Convención, incorporando elementos éticos muy necesarios para esta interpretación.
\end{abstract}

Palabras clave: discapacidad, investigación científica

The ethical and legal duty to prevent and reduce to the maximum the appearance of new disabilities of the elderly in Chile

\begin{abstract}
In this article we pretend to show the inconsistency between the recent Law 20.584 with the United Nations Convention about the Rights of Persons with Disabilities. Such persons, in certain stage of their disease, would not be able to participate in research because of their disability condition. We consider that this impossibility does not agree with article 25 of the Convention, which requires the States prevent and reduce to the maximum the appearance of new disabilities, included those that initiate in the elderly. The problem generated does not have only a legal perspective, but also produces an ethical conflict affecting the State, health care professionals, patients and their families. For this reason, we propose a harmonic and systematic interpretation of article 28 of Law 20.584 with the Convention, incorporating ethical elements necessary for this interpretation.
\end{abstract}

Key words: disability, scientific research

A obrigaçáo ética e jurídica de prevenir e reduzir ao máximo o aparecimento de novas descapacidades em pessoas idosas no Chile

Resumo: Neste trabalho pretendemos evidenciar a incongruência entre a recente Lei № 20.584 com a Convenção das Nações Unidas sobre Direitos das Pessoas com Descapacidade. Ditas pessoas, em determinado estágio de sua enfermidade, não poderão participar de pesquisas por sua condição de descapacitados. Estimamos que esta impossibilidade não condiz com o artigo 25 da Convenção, que exige aos Estados prevenir e reduzir ao máximo o aparecimento de novas descapacidades, incluídas as que ocorrem em pessoas idosas. A problemática que é gerada não só possui uma perspectiva legal, senão que também produz um conflito ético que afeta o Estado, os profissionais médicos, os pacientes e seus familiares. Por isso, propomos uma interpretação harmônica e sistemática do artigo 28 da Lei $\mathrm{N}^{\circ} 20.548$ com a Convenção, incorporando elementos éticos muito necessários para esta interpretação.

Palavras-chave: descapacidade, investigaçáo científica

\footnotetext{
${ }^{1}$ Universidad de Talca, Chile

Correspondencia: criveros@utalca.cl

${ }^{2}$ Universidad Finnis Terrae, Chile
} 


\section{Introducción}

La autodeterminación en materia de salud, es una manifestación de la autonomía de las personas e implica el reconocimiento a la libertad de los individuos $(1,2)$. Así, Díez, al referirse a este tema, nos indica que: "la autodeterminación en materia de salud concede una posición activa al paciente respecto de su integridad física y psíquica, ofreciéndole la posibilidad de intervenir en su proceso vital. Esta circunstancia afecta de forma directa a sus relaciones con el médico, obligando a éste a ofrecerle la información precisa para el ejercicio del derecho y a respetar (con ciertos límites) sus decisiones. Así pues, se trata de una reciente perspectiva de la relación paciente-médico que se manifiesta de forma creciente en el ámbito de la sanidad pública"(3). La Declaración de Lisboa de la Asociación Médica Mundial, ya en 1981 recalcaba como uno de los principios fundamentales, en materia de los derechos de los pacientes, el derecho a la autodeterminación. De este modo, se declaraba que: "el paciente tiene derecho a la autodeterminación y a tomar decisiones libremente en relación a su persona. El médico informará al paciente las consecuencias de su decisión. El paciente adulto mentalmente competente tiene derecho a dar o negar su consentimiento para cualquier examen, diagnóstico o terapia. El paciente tiene derecho a la información necesaria para tomar sus decisiones. El paciente debe entender claramente cuál es el propósito de todo examen o tratamiento y cuáles son las consecuencias de no dar su consentimiento. El paciente tiene derecho a negarse a participar en la investigación o enseñanza de la medicina"(4).

\section{Consentimiento informado(5)}

El artículo 14 de la Ley $\mathrm{N}^{\circ} 20.548$ se refiere al consentimiento informado del paciente ${ }^{3}$. La exigua doctrina que existía en esta materia en Chile, elogió la inclusión en la norma del rechazo a un tratamiento médico; sin embargo, ha criticado la falta de referencia a la suspensión o interrupción de un tratamiento médico ya en curso. De acuerdo a la historia fidedigna de la ley, debiese

\footnotetext{
${ }^{3}$ De este modo el artículo 14 de la Ley de Derechos del Paciente establece que "toda persona tiene derecho a otorgar o denegar su voluntad para someterse a cualquier procedimiento o tratamiento vinculado a su atención de salud, con las limitaciones establecidas en el artículo 16".
}

efectuarse una interpretación extensiva de la norma, esto es, considerando el rechazo en un sentido amplio que permitiría albergar las nociones de suspensión e interrupción. Al respecto Zúñiga señala: "así se dispone que es posible que una persona aquejada de una enfermedad incurable y en fase terminal, decida no seguir siendo sometida a tratamientos, dándose por supuesto que el derecho al consentimiento informado incluye el derecho a la interrupción del procedimiento médico. Sin embargo, no cabe duda que es una clara deficiencia de la nueva normativa que se obligue de entrada al intérprete a fundamentar y justificar la existencia de uno de los principales derechos de los pacientes a nivel comparado e internacional" (6).

En este sentido el artículo 11 de la Ley $\mathrm{N}^{\circ} 20.120$ también se refiere al consentimiento informado del paciente, Con todo, esta norma es clara al establecer la posibilidad del suspender una investigación, al indicar en el inciso 2 que: "Asimismo, deberá hacerse especial mención del derecho que tiene de no autorizar la investigación o de revocar su consentimiento en cualquier momento y por cualquier medio, sin que ello importe responsabilidad, sanción o pérdida de beneficio alguno". Por lo tanto, en el caso de una investigación científica, siempre es posible una suspensión de la participación en dicha investigación.

Las relaciones familiares al momento de enfrentar enfermedades y muerte se complejizan a tal extremo que para muchos ya hablar acerca de estos temas es un gran problema. Asimismo, aceptar la enfermedad de un ser querido no es tarea fácil, mucho menos concordar con la decisión de un padre, madre o hermano, de desechar un tratamiento médico, que incluso puede significar la muerte. Tampoco resulta fácil aceptar la interrupción de un tratamiento. Por ello, el legislador debió haber previsto esta situación y no parece razonable dejar tan importante materia a la voluntad del intérprete, es decir, se debió haber establecido claramente si se podía suspender un tratamiento en curso.

Incongruencias de la Ley 20.584 (artículo 28, inciso $1^{\circ}$ ) con la Convención Internacional sobre Derechos de las Personas con Discapacidad (artículo 25, letra b): La autodeterminación en la discapacidad 
El punto central de este trabajo es establecer el alcance del artículo 28 , inciso $1^{\circ}$ de la Ley $\mathrm{N}^{\circ}$ 20.548 , ya que impide que una persona con discapacidad psíquica o intelectual, que no pueda expresar su voluntad, pueda participar en una investigación científica(7). Una posible respuesta a esta disyuntiva podría estar en la Ley $\mathrm{N}^{\circ} 20.120$ y su respectivo reglamento; sin embargo, dicha ley no se refiere a las personas con discapacidad en ninguno de sus preceptos. Asimismo, el reglamento, por una modificación introducida en enero de 2013, se remite nuevamente a la Ley $\mathrm{N}^{\circ}$ 20.584. De esta manera, el artículo 12, inciso 2 del reglamento de la Ley No 20.120 prevé: "En relación a la investigación científica con sujetos con discapacidad psíquica o intelectual, se deberá tener en cuenta lo señalado en la ley $\mathrm{N}^{\circ} 20.584$ y su reglamentación pertinente, en cuanto corresponda”. La remisión a la Ley $\mathrm{N}^{\circ} 20.584$ nos devuelve al mismo punto de partida, esto es, el artículo 28 del mismo cuerpo legal.

El reglamento de la Ley $\mathrm{N}^{\circ} 20.584$ se refiere a dos aspectos esenciales: la entrega de información y la expresión de consentimiento informado en las atenciones de salud. Desde el artículo 1 al 9 se establecen normas relativas a la información. Posteriormente, desde el 10 al 13 se establecen situaciones relacionadas con el consentimiento informado. En su artículo 1, inciso 3, al referirse a la información que se entrega al paciente, dispone: "En los casos de personas afectadas de enfermedad mental, a que se refiere el párrafo $8^{\circ}$, del título II, de la ley No 20.584, sobre derechos de las personas con discapacidad psíquica o intelectual, se procederá en conformidad a las disposiciones del decreto No 570, de 1998, del Ministerio de Salud, Reglamento para Internación de las Personas con Enfermedades Mentales y Sobre los Establecimientos que la Proporcionan, o el que en el futuro lo reemplace”.

Inicialmente, se podría pensar que la normativa del decreto $\mathrm{N}^{\circ} 570$ de 1998 nos entrega una respuesta satisfactoria al alcance de la aplicación del artículo 28 de la Ley $\mathrm{N}^{\circ}$ 20.584. Sin embargo, la remisión que el reglamento efectúa al decreto $\mathrm{N}^{\circ} 570$ de 1998 dice relación con la entrega de información al paciente y no con su expresión del consentimiento informado. Por ello, solo se puede recurrir al decreto $\mathrm{N}^{\circ} 570$ de 1998 respecto de la entrega de información y no en relación con la manifestación del consentimiento informado. Por lo tanto, la única interpretación posible, dada la regulación que hemos analizado, es entender que esta norma del artículo 28 de la Ley $\mathrm{N}^{\circ} 20.584$ solo rige para los casos en que la investigación científica no tiene como objeto devolver la salud al paciente sin someterlo a un riesgo superior al que ya sufre.

Nos parece evidente que el artículo 28 de la Ley $\mathrm{N}^{\circ} 20.584$ no se condice plenamente con las disposiciones contenidas en la Convención Internacional sobre Derechos de las Personas con Discapacidad, a lo menos si se entiende en un sentido absoluto. Nos parece especialmente esclarecedor su artículo 25, que establece que "los Estados Partes reconocen que las personas con discapacidad tiene derecho a gozar del más alto nivel posible de salud sin discriminación por motivos de discapacidad (...) En particular los Estados Partes: b) Proporcionarán los servicios de salud que necesiten las personas con discapacidad específicamente como consecuencia de su discapacidad, incluidas la pronta detección e intervención, cuando proceda, y servicios destinados a prevenir y reducir al máximo la aparición de nuevas discapacidades, incluidos los niños y las niñas y las personas mayores".

No son pocas las convenciones y tratados que exigen un rol activo del Estado relacionado con la salud y nuevas tecnologías. Es factible preguntarse qué rol tiene el Estado y la sociedad chilena en esta materia. ¿Cuál es la responsabilidad o corresponsabilidad que le cabe ${ }^{4}$. ¿Es posible, en razón de la imposibilidad de expresar de forma actual la voluntad, negar a una persona discapacitada psíquica o intelectualmente la participación en una investigación científica? Jurídicamente podría ser adecuado, aunque nosotros rechazamos dicha interpretación, pero, ¿̇lo es desde un punto de vista ético?

\footnotetext{
${ }^{4}$ De este modo, la Declaración Universal sobre Bioética y Derechos Humanos, de 19 de octubre del 2005, en su artículo 2, establece que "los objetivos de la presente Declaración son: d) reconocer la importancia de la libertad de investigación científica y las repercusiones beneficiosas del desarrollo científico y tecnológico, destacando al mismo tiempo la necesidad de que esa investigación y los consiguientes adelantos se realicen en el marco de los principios éticos enunciados en esta Declaración y respeten la dignidad humana, los derechos humanos y las libertades fundamentales".
} 
Del marco de los tratados internacionales y de una adecuada interpretación de la legislación chilena se debe desprender la regulación del consentimiento para estas personas. Así, la referencia a la recuperación de la salud es reiterada en dichos instrumentos. De este modo, la Declaración de la Asociación Médica Mundial se refiere al consentimiento informado de los incapaces y a las personas que no pueden manifestar su consentimiento en sus numerales 24 y 26, respectivamente. Estas disposiciones establecen que el investigador, al no poder apelar al consentimiento informado de los incapaces, debe recurrir al consentimiento informado del representante legal, conforme a la ley vigente. En igual sentido, el artículo $6, \mathrm{~N}^{\circ} 3$ de la Declaración Universal sobre Bioética y Derechos Humanos establece la misma regla, al exigir la autorización del representante legal o de la autoridad establecida por la ley. A su vez, la Declaración de la Asociación Médica Mundial pone como condición para usar discapacitados que la investigación no se pueda dar en personas capaces y debe ser necesaria para promover la salud de los que padecen la enfermedad (numeral 24).

La falta de consentimiento por imposibilidad físi$\mathrm{ca} /$ mental también es un aspecto reiterado en los instrumentos internacionales sobre la materia. De este modo, la Declaración de la Asociación Médica Mundial establece que la investigación solo puede llevarse a cabo si la enfermedad es el motivo de la imposibilidad, y de dicho impedimento debe dejarse constancia en el respectivo protocolo experimental, que debe estar sujeto a un comité de evaluación. De igual forma, la Declaración Universal sobre Bioética y Derechos Humanos, en su artículo 7, señala que las personas carentes de capacidad para dar su consentimiento podrán someterse a investigación científica, estableciéndose los siguientes mecanismos concretos para proteger su dignidad: la autorización para investigar deberá obedecer a los intereses del paciente y sujetarse a la legislación nacional; pero además ella solo se puede producir en la medida que la investigación redunde directamente en provecho de la salud de la persona interesada.

Las Pautas Éticas Internacionales para la Investigación Biomédica en Seres Humanos son especialmente interesantes a este respecto, por cuanto, además del beneficio de la intervención, se refie- ren al riesgo. En la Pauta $N^{\circ} 9$ se señala que "si existe una justificación ética y científica para realizar una investigación con individuos incapaces de dar consentimiento informado, el riesgo de intervenciones propias de la investigación que no proporcionen la posibilidad de beneficio directo para el sujeto individual no debe ser mayor que el riesgo asociado a un examen médico o psicológico de rutina de tales personas". Incluso se establece un claro criterio que relaciona el riesgo con el mejoramiento de la salud al agregar en la misma disposición que "puede permitirse incrementos leves o menores por encima de tal riesgo cuando exista una fundamentación científica o médica superior para tales incrementos y cuando un comité de evaluación ética los haya aprobado". En los comentarios a la Pauta se afirma que los comités de evaluación son los destinados a determinar la relación riesgo-beneficio en los casos en que no sea posible recurrir al consentimiento informado.

La Pauta $\mathrm{N}^{\circ} 13$ se refiere específicamente a los adultos mayores, pero como personas vulnerables. A este respecto, en los comentarios a dicha Pauta se señala que "los adultos mayores son comúnmente considerados vulnerables. A medida que la edad avanza, las personas tienen mayor probabilidad de adquirir características que las definen como vulnerables. Pueden, por ejemplo, estar internadas o desarrollar varios grados de demencia. Resulta apropiado considerarlas vulnerables y tratarlas como tales solo cuando hayan adquirido esos atributos".

Por otra parte, los comités de ética, establecidos en los artículos 17 a 20 de la Ley $\mathrm{N}^{\circ} 20.584$, que tratan de la autonomía de las personas en su atención de salud, no tienen competencia para resolver este problema. Sin perjuicio de lo anterior, en la primera parte del artículo 17 de la ley, se establece que los comités de ética se deben pronunciar respeto de los casos en que "el profesional tratante tenga dudas acerca de la competencia de la persona”, con relación a una negativa a iniciar o continuar con un tratamiento que el facultativo estime que expone a graves daños a la salud o pone en riesgo de morir al paciente, en la medida en que dichos riesgos "serían evitables prudencialmente siguiendo los tratamientos indicados". Y, a su vez, el inciso $4^{\circ}$ del artículo precedente, dispone que "tanto la persona como cualquiera a su 
nombre podrán, si no se conformaren con la opinión del comité, solicitar a la Corte de Apelaciones del domicilio del actor la revisión del caso y la adopción de las medidas que estimen necesarias. Esta acción se tramitará de acuerdo con las normas del recurso establecido en el artículo 20 de la Constitución Política de la República". A pesar de que esta norma no se refiere a la posibilidad de que una persona enferma o un anciano, que no pueda dar lugar al consentimiento informado, se someta a una investigación médica que eventualmente le permita sanarse, creemos que ante el vacío normativo es posible recurrir a ella. Ya que el problema que se plantea no es un conflicto solo de carácter legal; en este punto el conflicto también tiene una dimensión ética importante. Es cuestionable, desde un punto de vista humano, quién debe decidir. La bioética se presenta como una herramienta esencial. Ella cuestiona la norma jurídica y tal como expresa Gorjón: "La bioética como herramienta social nos proporciona criterios para manejo de valores extrajurídicos que permitan una ductilidad de la norma para su valoración en la dicotomía del ser humano" (8). En este sentido, permite analizar la norma jurídica desde otras perspectivas, buscando el bienestar del paciente.

Así, consideramos que por imposición de la Convención Internacional sobre Derechos de las Personas con Discapacidad, puede aplicarse el artículo 17 , incisos $1^{\circ}$ y $4^{\circ}$ de la Ley $N^{\circ} 20.584$ por analogía al caso que estamos analizando, llevando esta cuestión por el facultativo del Comité de Ética necesariamente a la Corte de Apelaciones para que ella decida. A ello se debe agregar que una prohibición de participar en una investigación científica, para aquellas personas que estén discapacitadas psíquica o intelectualmente (y ello no siempre ocurre, pero en no pocas ocasiones con adultos mayores $(9)^{5}$ y especialmente con personas mayores de 80 años), en términos absolutos, constituye una transgresión a Convención y un cuestionamiento ético al Estado, ya que dicha Convención impone al Estado el deber de prevenir y reducir al máximo la aparición de nuevas

\footnotetext{
5 "Vivimos en sociedades cada vez más envejecidas (...) o también lo que autores como Daniel Callaham denomina 'la avalancha de los mayores', comporta y va a suponer importantes cambios a todos los niveles: económico, político, sanitario y social, con los consiguientes desafíos que en cada uno de estos campos se plantean, tanto para la familia, como para las sociedades en general".
}

discapacidades. Las personas que sufren una enfermedad degenerativa viven en muchas ocasiones un largo y lento proceso de deterioro físico y mental, y la negativa absoluta de permitirles someterse a estos tratamientos no compatibiliza la prerrogativa de la autodeterminación con los derechos del paciente. Debiese incluso - como sucede en el Derecho Comparado- permitírsele al juez indagar en la voluntad presunta del enfermo. Evidentemente, más concordante con la autodeterminación del paciente, que no es más que una manifestación de la dignidad de la persona.

Los derechos del paciente están determinados en la transcrita letra b) del artículo 25 de la Convención. Así, es un sinsentido que un paciente, por ejemplo, que ha padecido por más de quince años una enfermedad como el Alzheimer, no pueda participar actualmente en una investigación que pudiese mejorar o por lo menos no desmejorar su actual estado de salud, solo porque de manera actual no puede expresar su voluntad. Es muy cuestionable desde el punto de vista ético. Una interpretación mosaica o literal del el artículo 28 de la Ley No 20.584 trasgrediría la citada letra b) del artículo 25 de la Convención. Entendemos que la autodeterminación tiene como sustrato básico la libertad de los individuos; pero la protección a esta autodeterminación no puede significar un desmedro en el bienestar de las personas.

Desde el punto de vista ético, en general, es complejo para los médicos lograr saber si el paciente está o no en condiciones de decidir, para así obtener un consentimiento informado. La situación es más sencilla cuando estamos en presencia de un adulto. Existen otros casos en que los pacientes son menores, por lo tanto, actuarán los tutores o representantes legales. Se plantea naturalmente un conflicto de carácter ético cuando no se puede tratar a un paciente, porque no puede expresar su voluntad. El problema en muchísimos casos es delimitar la competencia que posee el enfermo, ya que en muchas situaciones los pacientes son competentes para tomar algunas decisiones y otras no. De esta forma indica Quintana: "Evaluar la competencia de una persona es un ejercicio complejo. Hay que evaluar si la persona es capaz de comunicarse y entender la información, si es capaz de razonar sobre las alternativas y si tiene valores para poder juzgar" (10). 
La protección de los individuos con alguna discapacidad de tipo psíquica o intelectual, y de adultos mayores que no pueden dar consentimiento informado debe significar acompañarlos, ya sea en su enfermedad o en el proceso de envejecimiento. Como ya se indicaba en líneas anteriores, no es un proceso sencillo, ni para el médico, ni para el paciente y tampoco para su entorno. El reconocimiento de sus limitaciones no puede llevar a ignorar sus decisiones o, lo que es peor, dejarlas fuera del ordenamiento jurídico $(11)^{6}$. En el caso de los adultos mayores, el proceso de envejecimiento no es un asunto estático, sino dinámico $(12)^{7}$. No asociamos la ancianidad, que en muchos casos implica un saludable proceso de envejecimiento, con un prejuiciado concepto de vejez, sinónimo de enfermedad y discapacidad $(13)^{8}$.

En casos en que los ancianos se encuentren saludables, el Estado está obligado, ética y jurídicamente, a buscar todas las medidas tendientes a su autodeterminación en relación con los tratamientos médicos, tanto sea para su aceptación

\footnotetext{
6 "Cuando se pierda la capacidad de comprensión y comunicación, la capacidad de razonar o deliberar, e incluso cuando se pierda la conciencia, no será posible actuar autónomamente. Por eso es necesario establecer la importancia de dejar estipulado, mediante documentos previos (testamentos vitales o voluntades anticipadas) y según la propia escala de valores vitales de cada uno, aquello que queremos que se haga con nosotros, la manera de que clínicamente se nos trate, cómo nos gustaría ser clínicamente tratados en el proceso de nuestra muerte, quién nos representará y sustituirá mejor, etc., cuando ya no podamos decidirlo por nosotros mismos".

7 "El envejecimiento es una realidad que también tiene su dinamismo moral, que requiere una Ética aplicada propia, es decir, una reflexión ética ajustada a situaciones existenciales diferentes, pero en un horizonte de convicciones morales básicas. De esta forma, el dinamismo del envejecimiento ya no será únicamente un proceso de declive biológico o aislamiento sociológico, sino un proceso moral mucho más complejo. No sólo como un proceso de ajuste existencial para la persona mayor, sino un proceso para poner a prueba todas las dimensiones de la ética social (familia, servicios sociales, sanitarios, administrativos)".

${ }^{8}$ En este sentido, Di Tullio señala que "el derrotero de nuestra existencia bien puede ir de la mano de la salud, haciéndonos vivir un envejecimiento normal y fisiológico - la senectud. Ahora bien, cuando el proceso de envejecimiento se relaciona con alguna enfermedad, se vuelve patológico y, en este caso, podemos referenciar que estamos ante un caso de senilidad. Por tanto, senectud y senilidad no son sinónimos, aunque vulgarmente se haga referencia a ésta última para aludir a personas ancianas, cuando en rigor correspondería hablar de personas senectas. Así, en este trabajo, aceptamos como válida la definición de la vejez como un estadio normal del desarrollo, dejando de lado el prejuicio que asocia "vejez a enfermedad", como si fueran una idéntica e inseparable condición. Tampoco corresponde identificar vejez con incapacidad ni con dependencia, las cuales pueden
} aparecer con el correr de los ańos, pero no necesariamente". como para su rechazo. Y lo más importante, el Derecho debe velar por el establecimiento de una regulación que no discrimine a las personas ancianas a través de las reglas del consentimiento informado. Por ello, no es válido distinguir solo si los ancianos pueden o no dar consentimiento informado, por cuanto, al tratarse de un consentimiento calificado, en la mayoría de los casos no cumplirán el estándar que se exige a una persona no vulnerable. En este punto reiteramos la idea de que la competencia de un paciente no es un tema sencillo de abordar. Precisamente, el ordenamiento jurídico debe optar por la salud del adulto mayor, pero estableciendo resguardos adecuados mediante la intervención del representante legal y del juez, considerando su voluntad presunta cuando ella pueda ser reconstruida. Esa es una respuesta ética adecuada. Solo de esta forma el ordenamiento jurídico se hace cargo de los ancianos, que van envejeciendo acompañados de una enfermedad, que va debilitando sus capacidades $(14)^{9}$.

En definitiva, entendemos que la normativa actual, relativa a los derechos de los pacientes, no ha sido consciente de la complejidad del tema, al prohibir la posibilidad de investigación científica para pacientes que no puedan expresar su voluntad. Dicha norma, tendiente a proteger a los discapacitados, actúa en muchas ocasiones como un límite para su bienestar, evidenciando la necesidad ética de flexibilizar tal prohibición para que se ajuste a las exigencias establecidas por la Convención Internacional de Derechos de la Personas con Discapacidad y las Declaraciones Internacionales precedentemente estudiadas. Nuevamente Gorjón: "La bioética tiene como finalidad el análisis racional de problemas morales ligados a la biomedicina y su vinculación con el ámbito del derecho y de las ciencias humanas, esta finalidad implica elaborar lineamientos éticos fundados en valores de la persona y en los derechos humanos, con bases racionales que derivan de una metodología científica”(8).

\footnotetext{
${ }^{9}$ Zurita explica la relación entre la edad y la capacidad de autogobernarse. "En verdad, si bien no puede decirse que la avanzada edad de una persona sea considerada como una concreta enfermedad, sí puede afirmarse que el anciano sufre toda una serie de limitaciones físicas y psíquicas precisamente por razón de aquellas.

Las limitaciones propias de la edad no dan lugar, necesariamente, a la incapacitación del anciano, puesto que habrá que atender (...) a su capacidad de autogobierno".
} 
Por ello, estimamos que esta norma de la Convención permite la investigación científica, es decir, que una persona enferma, estando con sus facultades mentales, puede decidir que, cuando su enfermedad vaya avanzando, sea sometida a investigación o un tratamiento experimental, que no existe al tiempo actual. Lo determinante es si dicha autorización incluye una investigación riesgosa, por cuanto los riesgos de efectos secundarios, que están presentes en estas investigaciones y tratamientos, no serán conocidos por el paciente. $\mathrm{Si}$ los riesgos son altos, creemos que se debe optar por la autorización judicial. En otras palabras, la norma, cuando establece que "ninguna persona con discapacidad psíquica o intelectual que no pueda expresar su voluntad podrá participar en una investigación científica”, solo se está refiriendo a los casos en que el paciente no ha prestado su autorización de forma personalísima. Así, aun cuando una persona nada haya señalado respecto de su participación en una investigación científica, cuando estaba en uso de sus facultades, ella podrá ser objeto de dicha investigación en la medida en que el juez lo autorice, a solicitud de un pariente próximo o de su representante legal. Nos inclinamos a favor de esta interpretación, por cuanto es la única compatible con el artículo 25 de la Convención de las Naciones Unidas sobre los derechos de las personas con discapacidad. Asimismo, nos parece que los principios constitucionales de la dignidad e igualdad ante la ley, consagrados en los artículos 1 y 19, No 2, de la Constitución chilena nos obligan a llegar a esta conclusión. Siguiendo a Figueroa, es posible sostener que: "el derecho humano a escoger la vida que cada uno desea llevar, el derecho a "hacer la vida" es lo que le da su dignidad intrínseca. Si este derecho a "hacer la vida" es, en verdad, un derecho a escoger valores, a vivir y a luchar por ellos, como ya dijimos, su ejercicio es una manifestación de dignidad. Este derecho a la vida digna se prolonga, como es obvio, hasta el día de la muerte. A la vida digna debe seguir una muerte igualmente digna(15)". La igualdad ante la ley exige que si una persona enferma determina que quiere someterse a un tratamiento o investigación lícita en torno a su enfermedad, pueda hacerlo, ya que no porque su discapacidad sea intelectual podría ser discriminado como si se tratara de una enfermedad degenerativa a la columna, por ejemplo.
El principio de la dignidad se relaciona con la no discriminación claramente en consideración a lo preceptuado en el preámbulo letra h) de la Convención ${ }^{10}$. De igual forma, el Mensaje de la Ley de Derechos del Paciente dispone que: "respetar la dignidad de las personas no es una mera afirmación declarativa o una bien fundada pretensión programática. Al contrario, ésta se traduce en formulaciones y hechos concretos, especialmente en el respeto por su libertad. Una sociedad democrática requiere avanzar en el desarrollo humano, una de cuyas expresiones es la ampliación de libertades, como la autonomía y control sobre el propio cuerpo y sobre las decisiones del entorno que conciernen a su integridad y al ejercicio de sus derechos". Este texto, aunque es solo programático, ubica la discapacidad en el contexto de la ancianidad. Así, no todas las decisiones podrán ser tomadas en un tiempo real; por ello, el reconocimiento a la voluntad anteriormente manifestada es vital para una verdadera aplicación de la autonomía y la libertad en los casos de discapacidad evolutiva.

\section{Conclusión}

En primer lugar, valoramos como muy positivo que a nuestro Derecho se haya incorporado una ley relativa a los derechos y deberes que tienen las personas en relación con acciones vinculadas a su atención en salud (Ley $\mathrm{N}^{\circ}$ 20.584). Esta normativa acertadamente considera los denominados derechos clásicos del paciente: consentimiento informado, el acceso a los registros médicos y el carácter confidencial de sus datos.

Sin embargo, hemos podido notar que, a poco andar, dicha normativa legal ha generado - a nuestro modo de ver- conflictos tanto con la Convención de las Naciones Unidas sobre los Derechos de las Personas con Discapacidad como con las diversas Declaraciones sobre ancianidad y discapacidad suscritas por Chile.

Existen muchas enfermedades que si bien en un comienzo no generan discapacidad, en el transcurso de tiempo van disminuyendo las facultades

\footnotetext{
${ }^{10}$ En este sentido, la parte considerativa de la Convención establece que "reconociendo también que la discriminación contra cualquier persona por razón de su discapacidad constituye una vulneración de la dignidad y el valor inherentes del ser humano".
} 
físicas y mentales a las personas que las padecen. Una interpretación con un preponderante contenido ético de las normas legales conduce a que la persona que padece por mucho tiempo una enfermedad pueda determinar si quiere o no participar en una investigación, y que incluso otros puedan tomar dicha decisión fundados en la recuperación de la salud de la persona de edad. No nos parece ni razonable ni adecuado exigir una voluntad en tiempo real, incluso nos inclinamos porque el juez - como sucede en el Derecho comparado-, en la mayoría de estos casos, tenga la facultad de establecer o reconstruir una especie de voluntad presunta del enfermo. También nos parece que en los casos en que no sea posible reconstruir dicha voluntad se pueda dar lugar a la participación en la investigación del anciano, pero sujeto al control del representante legal, la autorización judicial y del Comité Ético, quienes debe tomar en consideración el beneficio del paciente con relación al riesgo al que se le puede someter.

\section{Agradecimientos}

$\mathrm{Al}$ profesor Jochen Taupitz por su generosa ayuda en recolección de material de Derecho alemán. Asimismo, agradecemos a Cristina Albornoz por su colaboración en la revisión de aspectos formales de este trabajo. 


\section{Referencias}

1. Taupitz Jochen. Selbstbestimmungsrecht des Patienten: Aktuelle Kontroversen im deutschen Recht. En Dogan H, Mahmutoglu F, Namal A, (eds.) Türkisches Jahrbuch für Studien zu Ethik und Recht in der Medizin; 2008: 43.

2. Taupitz Jochen. Ethische und rechtliche Begrundung von Patientenautonomie und Patientenvertretung. En Patientenautonomie-Patientenvertretung. Begründung und Ansätze zu ihrer Verwiklichung, 2002: 34.

3. Díez Laura. La garantía institucional de la libertad del paciente. Revista de Bioética y Derecho, Barcelona, Facultad de Derecho Universidad de Barcelona, 2012; 25: 37.

4. Asamblea Médica Mundial. Declaración de Lisboa sobre los Derechos del Paciente. Adoptada por la 34a Asamblea Médica Mundial Lisboa, Portugal, septiembre/octubre 1981 y enmendada por la 47a Asamblea General Bali, Indonesia, septiembre 1995 y revisada su redacción en la 171a Sesión del Consejo. Santiago de Chile; 2005. Disponible en: http:// www.wma.net/es/30publications/10policies/14/ )Consultada el 7 de diciembre de 2012).

5. Cañete R, Guilhem D, Brito K. Consentimiento informado: algunas consideraciones actuales. Acta Bioethica 2012; 18(1): 121-127.

6. Zúñiga Alejandra. La nueva Ley de Derechos del Paciente: más dudas que certezas. Disponible en: www.diarioconstitucional.cl (Consultado el 19 de noviembre de 2012).

7. Steiner T, Walter-Sack I, Taupitz J, Hacke W, Strowitzki T. Ethische und juristische Aspekte beim Einchluss nicht einwilligungsfähiger Patienten in Akuttherapie-Studien. Dtsch MEd Wochenschr 2008; 133: 787-792.

8. Gorjón Gómez Gabriel De Jesús. Bioética y propiedad intelectual. Un enfoque concomitante de facto y jure. Letras Jurídicas 2013; 17: 1-27.

9. García Ferez José. La bioética ante la enfermedad de Alzheimer. Desclée De Brouwer; 2005: 25.

10. Quintana Trías Octavi. Bioética y Consentimiento Informado. En Casado González María. (ed.) Materiales de bioética $y$ derecho. Barcelona: Cedecs; 1996: 157-170

11. García Férez José. Bioética y Personas Mayores. Madrid; 2003. (Consultado el 31 de marzo de 2003) Disponible en http://www.imsersomayores.csic.es/portal/senileg/documentos/garcia-bioetica-01.pdf

12. Moratalla Agustín Domingo. Claves para una ética de la ancianidad. Bioètica \& debat: Tribuna abierta del Institut Borja de Bioètica 2000; 20: 1-4.

13. Di Tullio Rosana. La evolución legislativa y jurisprudencial en materia de salud mental referida a la vejez. Perspectiva en el Derecho Proyectado. Revista Interdisciplinaria de Doctrina y Jurisprudencia 2012; 56: 23-37.

14. Zurita Isabel. La incapacitación del anciano. En Zurita Isabel. Protección civil de la ancianidad. Dykinson; 2004.

15. 15. Figueroa Yáńez Gonzalo. Algunas consideraciones sobre la vejez y la muerte ante el Derecho Civil. Estudios de Derecho Civil III. Jornadas Nacionales de Derecho Civil. Valparaíso; 2007: 23-47.

Recibido: 10 de diciembre de 2013

Aceptado: 16 de abril de 2014 